\title{
The Effect of a Pressurised Mid-Span Gas Seal on Fluid-Induced Rotor Instability
}

\author{
John J. Yu ${ }^{\dagger}$, Paul Goldman, Donald E. Bently and John S. Jacob \\ Bently Rotor Dynamics Research Corporation, 1631 Bently Parkway South, Minden, Nevada 89423, USA
}

(Received 20 June 2001; accepted 6 September 2001)

\begin{abstract}
Fluid-induced instability of a rotor-bearing system occurs frequently in rotating machinery. This involves two types of instability, i.e., fluid whirl and whip. Whirl is a predominately rigid-body mode and tracks at around one-half shaft running speed. Its threshold speed can be arbitrarily raised by using externally-pressurised fluid bearings, which allow for adjustable pressure for high stiffness. Whip is mainly a rotor-bending mode and locks into the rotor natural frequency. It appears when increases in bearing stiffness no longer influence the instability threshold. As demonstrated both analytically and experimentally in this paper, however, use of an externallypressurised gas seal in the mid-span of the rotor can successfully eliminate whip. Its threshold speed can be greatly raised beyond running speed by adding the mid-span seal, to nearby the antinode of the rotor-bending mode. An experimental rig was operated at speeds up to $10,000 \mathrm{rpm}$, far above the rotor natural frequency of less than $2000 \mathrm{rpm}$ without the seal. The gas supply pressure could be adjusted to yield the stiffness required for eliminating whip, which would otherwise appear even with an externally pressurised fluid bearing at the shaft end. This approach encourages utilisation of existing process gas as the supporting medium to eliminate fluidinduced instability.
\end{abstract}

${ }^{\dagger}$ Member of the International Institute of Acoustics and Vibration (IIAV)

\section{INTRODUCTION}

Fluid whirl and whip have been known as major instability problems in rotors supported by fluid-lubricated bearings. Black $^{\mathbf{1 , 2}}$ made significant contributions to the knowledge of fluid/structure interaction problems in rotating machines. Crandall ${ }^{3}$ also demonstrated whirl and whip problems by using Sommerfeld solutions due to Robertson ${ }^{4}$. The understanding of the fluid whirl and whip has been clear since a fluid force model was developed by Bently and Muszynska, ${ }^{\mathbf{5},}$. The description of the physical situation is simplified in this model as a cylinder rotating inside a stationary cylinder, with a relatively small clearance between them filled with fluid. The rotating cylinder drags the fluid into rotation. The fluid in motion generates forces that in feedback act at the rotating cylinder and drag it into lateral vibrations.

This fluid model fully interprets instability of rotors supported by isotropic bearings using a key parameter $\lambda$ (fluid circumferential average velocity ratio), a function of rotor eccentricity. The product $\lambda \Omega(\Omega$ is the shaft rotational speed) represents the angular velocity at which the fluid force rotates, as demonstrated both analytically and experimentally. ${ }^{\mathbf{5} 6}$ It has been shown that such a simplified model for an isotropic bearing, if neglecting fluid inertia effects, can be given in a fixed reference frame by

$$
-\boldsymbol{F}_{f}=D \dot{\boldsymbol{r}}+(K-j \lambda \Omega D) \boldsymbol{r},
$$

where $\boldsymbol{F}_{f}$ represents the fluid force, and $K$ and $D$ are the fluid stiffness and damping. The rotor lateral response is described by the vector of displacement $\boldsymbol{r}=x+j y$ ( $x$ and $y$ are horizontal and vertical displacements, respectively, and $j=\sqrt{-1}$ ). Equation (1) represents a fluid force rotating forward at average speed $\lambda \Omega$ with stiffness $K$ and damping $D$. The relation between these dynamic stiffness parameters such as $K, D$, and $\lambda$ and bearing coefficients can be established easily, even for anisotropic bearings with additional parameters. ${ }^{7,8}$ For simplicity, an isotropic bearing model in Eq. (1) is used for oil and gas bearings/seals in this paper. Note that Eq. (1) is equivalent to the following expression in terms of classical damping and stiffness coefficients

$$
-\boldsymbol{F}_{f}=-\left\{\begin{array}{c}
F_{x} \\
F_{y}
\end{array}\right\}=\left[\begin{array}{cc}
D & 0 \\
0 & D
\end{array}\right]\left\{\begin{array}{l}
\dot{x} \\
\dot{y}
\end{array}\right\}+\left[\begin{array}{cc}
K & \lambda \Omega D \\
-\lambda \Omega D & K
\end{array}\right]\left\{\begin{array}{l}
x \\
y
\end{array}\right\} .
$$

Use of externally-pressurised fluid bearings such as the ServoFluid $^{\mathrm{TM}}$ Control Bearing (SFCB) ${ }^{9}$ located at the shaft ends can successfully eliminate fluid whirl, a predominately rigid-body mode. The oil bearing stiffness $K$ as described above, which has primary influence on the whirl instability, can be arbitrarily increased by raising the fluid supply pressure. When the whirl threshold speed reaches beyond the otherwise whirl speed range, the whirling motion can be completely avoided. However, as the oil bearing becomes stiffer compared with shaft stiffness, oil whip might appear. This is often seen when the shaft speed exceeds twice the rotor natural frequency.

In order to control whip, one must decrease the fluid circumferential average velocity ratio $\lambda$ and increase the overall stiffness of the system. Some anti-swirl devices ${ }^{10,11}$ have been introduced to decrease $\lambda$. However, only a few efforts have been made to increase the overall system stiffness. The work described here is mainly concerned with effective means to increase the overall stiffness required to deter whip by the use of an attached gas seal.

In this paper the effect of a mid-span gas seal on the instability of whip, a predominately bending mode, is discussed in detail. A simplified mathematical model consisting of oil 